\title{
THE VENOUS MANIFESTATIONS OF PULSE WAVE ENCEPHALOPATHY: WINDKESSEL DYSFUNCTION IN NORMAL AGING AND SENILE DEMENTIA
}

Grant A Bateman MBBS FRANZCR ${ }^{1,}$ Christopher R Levi MBBS FRACP ${ }^{2}$,

Peter Schofield MD FRACP ${ }^{3}$, Yang Wang $\mathrm{PhD}^{2}$, Elizabeth C Lovett B.A. (Psych) ${ }^{2}$

1. Medical Imaging, John Hunter Hospital, Newcastle, Australia

2. Clinical Neurosciences Program, Hunter Medical Research Institute, Newcastle, Australia

3. Neuropsychiatry Unit, James Fletcher Hospital, Newcastle, Australia

Running title: Venous pulsation in dementia

$\begin{array}{cl}\text { Word count- paper } & 5560 \text { words } \\ \text { - abstract } & 232 \text { words } \\ \text { - title } & 100 \text { characters }\end{array}$

Correspondence: Dr Grant Bateman

Department of Medical Imaging

John Hunter Hospital

Locked Bag 1

Newcastle Region Mail Center

2310 Australia

Phone: 61249213414

Fax : 61249213428

Email: grant.bateman@hnehealth.nsw.gov.au 


\section{ABSTRACT}

Introduction: Cerebral arterial, venous and CSF pulsations are closely coupled and this produces pulsation dampening or the windkessel effect. Normal pressure hydrocephalus is a manifestation of the breakdown of this windkessel effect with altered CSF and venous pulsations being noted. The aim of this paper is to show that dysfunction of the windkessel mechanism is also a component of normal aging and senile dementia.

Methods: 24 patients were classified as either early senile dementia of Alzheimer's type (SDAT) or vascular dementia (VaD) and were compared with 12 age matched noncognitively impaired subjects.12 normal young individuals (NY) were compared to the normal aging group (NE). MRI flow quantification was used to measure the non-pulsatile and pulsatile components of blood flow as well as the pulsation at the tentorial incisura. Results: With normal aging blood flow decreases but arterial pulsations increase in volume by $49 \%(p=0.003)$. The CSF vented via the tentorial incisura does not change significantly with age and therefore increased venous pulsation is necessary. In VaD the arterial pulse volume was higher by $24 \%$ and the straight sinus pulsation increased by $57 \%$ compared to normal aging ( $p=0.05$ and 0.03 respectively). In SDAT the total venous pulsation volumes were similar to normal aging but there was less basal sinus pulsation.

Conclusion: Normal aging, SDAT and $\mathrm{VaD}$ are associated with alterations in venous pulsation due to a breakdown of the windkessel effect. 
KEYWORDS

Alzheimer's disease, cerebral blood flow, cerebrospinal fluid dynamics, vascular dementia.

\section{INTRODUCTION}

Arterial blood flow is pulsatile but the capillary bed of the brain requires non pulsatile continuous flow. Greitz was the first to show that intracranial arterial pulsations are dampened by shifting an equivalent volume of CSF and venous blood out of the cranial cavity [1]. Egnor et al building on the work of Greitz, modeled the dynamics of the cerebral vascular tree and CSF spaces and showed that the arterial pulsation and the CSF pulsation through the foramen magnum are very closely coupled [2]. So closely coupled that the CSF oscillations are in a state of resonance with the arterial pulsations. This state of resonance is not a trivial matter, as it requires the almost exact matching of the elastic and inertial reactance of the craniospinal system. The beneficial effect of this resonance is that a dynamic oscillator so configured will maximally dampen the arterial pulsations before the capillary bed by removing pulsation energy in systole and re applying it to the arterial tree in diastole. The dampening of pulsations is the windkessel effect. The impedance of such a system is at a minimum when the CSF and arterial pulsations are equal in amplitude with a zero phase lag between them. Under these circumstances all of the pulsation energy would be conserved and it would be unnecessary for any pulsation to exit the system via the veins. However the ideal windkessel effect does not appear to operate in the brain because there is venous out flow pulsation. The Monroe-Kellie doctrine states that an increase in arterial volume can only be accommodated by decreasing the CSF or venous volume (the brain volume being 
constant). Therefore if the windkessel effect fails, it would be expected that a greater percentage of arterial pulsation would be directed to compressing cortical veins. This cortical vein compression dissipates and allows dampening of the arterial pulsation. The main difference between the resonant dampening occurring via CSF motion and nonresonant dampening from venous compression is that the venous pulsation energy is not recycled but lost to the venous outflow.

A study of the hydrodynamics of normal pressure hydrocephalus (NPH) showed reductions in blood flow and craniospinal compliance, elevated brain and aqueduct pulsations and suggested altered venous compression and elevated venous pressure [3]. Egnor et al reduced the craniospinal compliance in their model of brain pulsations to simulate hydrocephalus and the model predicted a decrease in cerebral blood flow, an increase in arterial pulse pressure, increased choroid plexus pulsations and aqueduct pulsation resulting in dilation of the ventricles. In addition the model predicted there would be an elevation in outflow venous pressure despite the overall reduced inflow. The elevated venous pressure would reduce the CSF-sagittal sinus pressure gradient and bring about CSF malabsorption [2]. Using a non invasive surrogate measure of venous pressure based on estimating the venous collateral flow, an elevation in venous pressure has been suggested in both the obstructed and communicating forms of chronic hydrocephalus in the elderly as predicted by the model [4].

It has been noted that both senile dementia of Alzheimer's type (SDAT) and vascular dementia (VaD) are closely associated with NPH. A study into normal pressure hydrocephalus used a group with mixed dementia as controls and noted there were altered pulsations in this group as well as the NPH group [3]. Further, leukoaraiosis is a major component of $\mathrm{VaD}$ and it has been shown there are arterial and venous pulsation abnormalities associated with this disorder [5]. A hypothesis was developed linking 
dementia with hydrocephalus and altered pulsation propagation [6]. It was hypothesized that normal aging and senile dementia may be manifestations of a breakdown in the windkessel effect either by providing too large an arterial pulsation to be dampened, too small a compliance in the outflow pathways to allow dampening or a combination of both. It was suggested a cerebral parenchymal derangement could ensue from the abnormal pulsation stresses if dampening failed. The term pulse wave encephalopathy was coined to describe this effect. Since this hypothesis was published several groups have been actively researching the effect of windkessel dysfunction on arterial flow and capillary pulsation [7-9]. However, there has been little interest in the venous manifestations of windkessel dysfunction. This paper discusses the CSF and venous pulsation effects which occur with normal aging and senile dementia to define the venous windkessel dysfunction associated with these processes.

\section{METHODS}

\section{Subjects}

Twenty-four patients were recruited from neurovascular and memory clinics in a tertiary referral hospital setting. Cognitively impaired patients were classified as having either probable Senile dementia of Alzheimer's type (SDAT) or probable vascular dementia on the basis of clinical features, CT and/or MR brain imaging, Hachinski score and bedside neuropsychological testing. Dementia or cognitive impairment was confirmed with formal neuropsychological testing using NINDS- DSM IV criteria. Finally, a consensus meeting involving a neurologist skilled in cerebro-vascular disease (CL), a neurologist skilled in memory disorders (PS) and a neuro-psychologist (EL) was used to designate patients as early vascular dementia/cognitive impairment or early Alzheimer's disease/ cognitive impairment blinded to the MR flow data. In order to 
obtain informed consent and to study patients early in their disease, patients with a mini mental state examination less than 17 were excluded. The SDAT patient group consisted of 8 females and 4 males, mean age $76 \pm 4$ (SD) years. The SDAT group contained 10 patients with confirmed early dementia and two with significant cognitive impairment. The vascular dementia patient group consisted of 5 females and 7 males, mean age $70 \pm 11$ (SD) years. Eight of these patients had confirmed early dementia and 4 had significant cognitive impairment. The normal elderly (NE) control group was selected from the nondemented spouses of patients enrolled in the study and healthy volunteers, with each patient undergoing mini mental state examination and then formal neuropsychological testing. The NE control group consisted of 6 females and 6 males mean age $70 \pm 5$ (SD) years. A second control group i.e. the normal young (NY) was recruited from healthy volunteers and consisted of 8 females and 4 males aged 25 \pm 12 (SD). Informed consent was obtained from all patients and the hospital ethics committee approved the study protocol.

\section{MR and Analysis}

All patients were imaged on a $1.5 \mathrm{~T}$ superconducting magnet (Magnetom Vision; Seimens, Erlangen Germany) with the reporting radiologist (GB) blinded to the clinical details. For the blood flow measurements a retrospectively cardiac gated phase contrast flow quantification sequence was used with a TR of $29 \mathrm{~ms}$, TE $7 \mathrm{~ms}$, flip angle $30^{\circ}$, slice thickness of $6 \mathrm{~mm}$, matrix 192x512, FOV 200 and a single excitation. For the arterial measurements a velocity encoding (venc) value of $75 \mathrm{~cm} / \mathrm{sec}$ was used and a plane of section was selected to intersect the basilar artery and the cavernous portion of the internal carotid arteries at the skull base, as per the literature [5]. A venc value of 40 $\mathrm{cm} / \mathrm{sec}$ was used to measure the superior sagittal sinus (SSS) and straight sinus (ST) flow 
with the slice angled to intersect the SSS about $2 \mathrm{~cm}$ above the torcular and pass through the midpoint of the ST. A TR of 100, TE of 12 and a venc value of $10 \mathrm{~cm} / \mathrm{sec}$ was used the measure the flow in the region of the tentorial incisura with the slice placed perpendicular to the mid portion of the midbrain. Regions of interest were placed around the carotid arteries and basilar artery in each patient and these flows summed to give the total supratentorial inflow. The SSS and ST venous flows were obtained from the regions of interest placed around these vessels and the percentage of the arterial inflow that each vein represented for each patient was calculated. The incisural flow was obtained from a region of interest encompassing the cisterna ambiens, interpeduncular cistern, the mid brain and aqueduct. Care was taken to exclude aliasing by retrospectively manipulating the base lines of each resultant graph giving, for example, an effective upper arterial flow limit of $150 \mathrm{~cm} / \mathrm{sec}$. The MR flow quantification sequence used in this study is a standard acquisition protocol and is available on most commercial scanners.

The arterial pulse volume represents the degree to which the arterial tree expands in systole and is calculated from the graphs obtained from each carotid and basilar artery. The mean blood flow velocity for each artery for the entire heartbeat was subtracted from the mean blood flow velocity for the period of systole for the same artery, giving the mean increase in flow velocity over systole for that vessel. This value when multiplied by the time taken for systole to occur and the crossectional area of the vessel (region of interest) gives the volume of expansion of that vessel in systole. This method is similar to one previously published [5]. The addition of the value of vessel expansion obtained for both carotids and the basilar arteries gave the arterial pulse volume. The sagittal sinus and straight sinus pulsations were similarly defined as the increase in flow occurring above the mean flow in systole. The incisural pulse represents the total volume vented through the tentorial incisura and was obtained for each patient by a similar process to the 
arterial pulse volume. By obtaining the mean flow velocity directed inferiorly towards the feet in systole and multiplying this result by the time taken for the negative flow and the crossectional area of the region of interest, the pulse volume was obtained. The basilar sinus venous pulsation was estimated as the arterial pulse volume minus the sagittal sinus, straight sinus and incisural pulsations.

Mean and standard deviations were obtained for each group of patients. Differences between the groups were tested using a nonpaired $T$ test with a $\mathrm{p}$ value of less than 0.05 used to indicate significance.

\section{RESULTS}

The non-pulsatile hemodynamic findings are summarized in table one, whilst the pulsatile findings are summarized in table two. The arterial flow and pulsation data for the elderly patients has been previously published [9] but the remaining data including the venous flow and pulsation data, the incisural pulsation data as well as the arterial data for the young patients has not previously been published.

Compared to the normal young group (NY) the normal elderly group (NE) showed a $21 \%$ reduction in arterial inflow $(\mathrm{p}=0.003)$. A flow reduction of approximately $23 \%$ in the sagittal sinus $(\mathrm{p}=0.007)$ and $25 \%$ in the straight sinus $(\mathrm{p}=0.02)$ was noted indicating that a similar reduction in blood flow was occurring in each of the venous territories with aging. Despite the reduction in mean flow, the pulsatile components of arterial flow increased with aging by $49 \%(\mathrm{p}=0.003)$. The pulsation leaving by the incisura was not significantly different with aging. The venous pulsations were increased by $55 \%$ in the sagittal sinus $(p=0.02), 75 \%$ in the straight sinus $(p=0.0003)$ and calculated to be increased by $56 \%$ in the remaining basal sinus outflow $(\mathrm{p}=0.0002)$. 
The average mini mental state examination result for the normal elderly group was $29 / 30 \pm 2$, the SDAT group $23 / 30 \pm 4$ ( $p=0.0003$ compared to the controls) and the VaD group $26 / 30 \pm 2$ ( $p=0.005$ compared to the controls). The cerebral inflow in the SDAT patients was $18 \%$ less than the NE group $(\mathrm{p}=0.01)$ with the $\mathrm{VaD}$ group being not significantly different to the normal elderly. The SSS flow for the SDAT group was reduced by $16 \%(p=0.03)$ compared to the normal elderly but the SSS flow for VaD and all the ST flows were not significantly different. As a percentage of the inflow the SSS flow was reduced by $6 \%$ in $\mathrm{VaD}(\mathrm{p}=0.05)$ with the other percentage outflows being not significantly different.

The arterial pulse in $\mathrm{VaD}$ was $24 \%$ higher than the normal elderly $(\mathrm{p}=0.05)$. The incisura pulse was $64 \%$ higher for the $\mathrm{VaD}$ patients compared with the normal elderly $(p=0.001)$ and the ST pulse was $75 \%$ larger $(p=0.03)$ with the SSS and basal sinus pulsations being not significantly different. The arterial, SSS, ST and incisural pulses for the SDAT patients were not significantly different to normal aging but the basal sinus pulsation was calculated to be reduced by $39 \%(p=0.01)$.

\section{DISCUSSION}

The volume of blood passing into the brain has been measured using MR flow quantification. The MR sequence works by measuring the phase angle change induced in the spins of the protons as they pass through the magnetic field of the scanner in the slice selected. The phase change is proportional to the velocity. The sequence is gated to the heartbeat and approximately 200 heartbeats are combined giving on average 24segmented measurements of velocity per $\mathrm{R}$ to $\mathrm{R}$ interval. Because the velocity across the entire heartbeat can be averaged for any pixel, the volume passing through this pixel can be measured by multiplying the velocity obtained by the area of the pixel. This technique 
has been validated both in vitro and in vivo and found to have error rates in the order of $5 \%$ with a tendency to slight underestimation of flow, the intra and interobserver variability is described as low to negligible [10-12]. The blood flow volume passing through a vessel can be measured in millilitres per minute $(\mathrm{ml} / \mathrm{min})$ by summing the volumes of blood passing for all the pixels in a defined area of interest, which encompasses the vessel [13]. Summing the volumes obtained for each carotid artery and the basilar artery gives the cerebral blood inflow for the supratentorial cavity. The total inflow into the supratentorial cavity must be equivalent to the outflow, however it is noted that the SSS and ST represent only about $60 \%$ of the inflow. The remainder of the outflow occurs via accessory venous channels and the basal venous sinuses, which drain the inferior frontal, temporal and occipital lobes.

The cerebral arterial blood flow pulsates, with more blood entering the vessels over and above the mean flow in systole and less in diastole. It has been pointed out that the blood leaving the arterial tree and entering the capillary bed normally does not pulsate [14] and because fluids are incompressible, the extra blood flowing into the arterial tree in systole must be accommodated by an expansion of the volume of the arterial tree. The supratentorial arterial pulsation has been measured by effectively integrating the area under the arterial blood flow curves in systole. The Monroe-Kellie doctrine states that CSF and blood are incompressible so either shifting CSF and/or compressing the venous structures must accommodate this arterial pulsation. The total volume of the CSF pulse (plus a small component of brainstem movement) has been directly measured at the tentorial incisura. In addition, the volume of venous blood displaced from the SSS and ST sinuses have been directly measured. Therefore, in order to satisfy the Monroe-Kellie doctrine the remaining pulsation volume must occur in the accessory and basal sinuses 
and can be estimated by subtracting the known outflow pulsations from the inflow pulsation.

\section{Windkessel dysfunction in normal aging}

From the tables it is noted that normal aging is associated with a reduction in mean arterial flow. The pulsatile components however, increase at a rate faster than the mean flow reduction. The pulse pressure for this cohort of normal elderly patients was 67 $\mathrm{mmHg}$ [9] compared to an expected figure of $40 \mathrm{mmHg}$ in normal young giving a significant increase in arterial pulse volume with aging [15] of close to 50\%. Pulsatile flow is a manifestation of the energy stored in the form of the pulse pressure and must be dampened. As previously discussed, dampening occurs by shifting CSF and venous blood, i.e. the windkessel effect. Theoretically, pulse dampening could be disrupted by either too large an input (large arterial pulsations) or too low a compliance in the output (cortical veins or vertebral canal being too stiff). A 50\% increase in arterial pulsation with aging would certainly be at risk of over stressing the system. In addition the compliance of the spinal canal and therefore the pulsation at the foramen magnum decreases with age by about $26 \%$ [16], therefore despite the increased overall arterial pulsation (which represents the input into the system) the volume change through the incisura does not change with aging reflecting the relative reduction in compliance in the spinal canal. Similarly, Czosnyka et al found that with aging there is an "increase in the elastic coefficient of the brain (decreased compliance) and a reduction in the cerebrospinal compensation reserve indicating the brain becomes stiffer and the CSF space less compliant with age" [17].

If the input is too large and one of the outputs is limited (incisural pulsation) the other outputs (i.e. the venous pulsation) must be increased to accommodate this. Two venous territories have been sampled to see how these increased pulsations are distributed 
i.e. the superficial venous system via the superior sagittal sinus (SSS) and the deep venous system via the straight sinus (ST) with the remainder of the pulsation being calculated as the basal sinus pulsation. It is noted that a significant increase in pulsation volume of at least $50 \%$ occurs in each venous territory with normal aging. Kim et al measured the SSS and ST pulsatility indices in a normal cohort of mean age 30 years and found pulsatilities of 0.33 and 0.28 respectively [18] whilst an older normal cohort of mean age 42 years had SSS and ST pulsatilities of 0.47 and 0.45 respectively [5] suggesting that venous pulsatility increases throughout life. Pulsatility indicies are relative measures of pulsation, where as the pulse volumes as used here, are absolute measures of pulsation and allow easier comparisons across patient groups.

The $50 \%$ increase in the arterial pulsation caused a percentage increase in pulsation of $55 \%$ in the SSS compared to $75 \%$ in the ST and $56 \%$ in the basal sinus pulsation ( $\mathrm{p}=0.02,0.0003$ and 0.0002 respectively). As expected, all else being equal, if the relative venous compliances of the three territories are the same with respect to each other, then it would be expected the pulsation distribution should be roughly equal (there being no significant change in the percentage of the mean flow from each territory with aging). In summary, normal aging is associated with elevated arterial pulsations to be dampened but the CSF flow to the spinal canal is limited. Of necessity, a much greater pulsation must pass to the venous outflow.

\section{Windkessel dysfunction in senile dementia}

With the development of SDAT there is a further reduction in arterial inflow compared to normal aging but the pulsatile components of flow are increased, therefore the total arterial pulsation is not significantly different to that of the normal aging group. The incisural pulsation is also not significantly different to normal aging giving a similar venous total pulsation. Although the total venous pulsation is similar to normal aging, the 
pulsation distribution is different. In normal aging the pulsation is directed to all of the sinuses equally but in SDAT the amount directed to the basal sinuses is reduced by $39 \%$ compared to normal aging $(\mathrm{p}=0.01)$. Similar to the discussion regarding the pulsation distribution above, this suggests that the inferior cortical veins draining into the basal sinuses must be relatively less compliant than the remainder of the venous territories for this to occur.

The cohort with early $\mathrm{VaD}$ did not show a reduction in arterial inflow. Similarly, Patankar et al showed no correlation between cerebral blood flow and leukoaraiosis (which is a major component of $\mathrm{VaD}$ ) [19]. There was a significant increase in the total arterial pulsation of $24 \%$ over normal aging $(\mathrm{p}=0.05)$ but the sagittal sinus pulsation was not increased. In this instance the pulsation redistribution was preferentially to the ST territory (a 57\% increase over normal aging $\mathrm{p}=0.03$ ) and the incisura (a $64 \%$ increase in pulsation volume over normal aging $\mathrm{p}=0.001)$.

\section{The cause of the venous flow and pulsation redistributions in senile dementia}

With normal aging comes an increase in CSF pulsations, which are directed to the entire venous outflow. With the development of SDAT the basal sinus pulsation is reduced in real terms. If the reduced compliance in the basal sinuses were due to an elevated basal venous pressure then the mean blood flow from these sinuses would be expected to be reduced but table one suggests this is not the case. In SDAT there is an increase in fibroblastic activity in the CSF and an increase in fibrosis of the arachnoid granulations [20] but to our knowledge no investigation into of the state of the basal veins has been performed.

In $\mathrm{VaD}$ there is an increase in total pulsations but again the SSS pulsation did not increase. In this instance (unlike in SDAT) the redistribution may be due to a global elevation in pressure of the entire SSS system including its cortical veins. Egnor et al 
have indicated that windkessel dysfunction will increase venous pressure in NPH [2]. An increase in SSS pressure has been shown to be associated with a reduction in the SSS venous return compared to the arterial inflow because of increased collateral venous flow [4]. Thus, the percent venous return has been developed as a surrogate marker of venous pressure. In NPH there is a 9\% reduction in the percent venous return $(p=0.04)$ [4] in $\mathrm{VaD}$ there is a $6 \%$ reduction in the percent venous return $(\mathrm{p}=0.05)$ suggesting a pressure elevation may occur in $\mathrm{VaD}$ which is somewhat less than that in NPH. Elevations in the SSS pressure will elevate the pressure in the superior cortical veins making them more resistant to compression and also reduce CSF reabsorption through the arachnoid granulations. It has been shown that there is a functional dichotomy between the deep and superficial drainage systems in NPH with lower pressures in the deep system compared to the superficial system [4] and this may promote the pulsation redirection to the deep system as shown in $\mathrm{VaD}$.

\section{Correlations between pulsation redistribution and pathology}

There is evidence of both an increased input into the dampening mechanism and reduced dampening capacity with aging and senile dementia but is this of significance? In SDAT there is stiffening of the intracranial arterial tree. This may render the efficiency of dampening of the arterial pulse pressure ineffective and increase the pulse pressure within the capillary bed $[1,9]$. In the basal venous territory there is also a suggested decrease in venous compliance indicating that capillary pulsations originating in the base of the brain will not easily propagate out of the capillaries and into the venous outflow. This would maximize capillary pulsation and brain pulsation at the expense of venous pulsation in the basal portions of the brain including the temporal lobes. Uftring et al have expressed similar findings regarding the compliance of the brain in AD stating; "Normal aging patients and Alzheimer's patients have a tendency for vascular pulsations to cause 
increased spinal cord oscillations and reduced CSF oscillations compared to young adults with the data suggesting that the $\mathrm{AD}$ patients changes are of larger amplitude than normal aged patients. This suggested there may be a decrease in compliance in the brain" [21]. It is interesting to note that the capillaries in $\mathrm{AD}$ have pathological changes of coiling and beading as well as evidence of basement membrane disruption [22-24]. There is also evidence of blood brain barrier breakdown in normal aging, SDAT and VaD [25]. Is there a causative link between capillary pulsation and capillary disruption in SDAT?

In $\mathrm{VaD}$, on the other hand, the arteriolar compliance may be elevated. The pulse pressure on the arterial side should be well dampened before the capillary bed, but at the expense of increased kinetic energy in the form of arterial wall recoil [9]. The pathology of $\mathrm{VaD}$ includes dilatation of the arteriolar perivascular spaces (etat crible) as well as white matter disease [26]. The elevated deposition of kinetic energy into the perivascular spaces may account for the etat crible noted pathologically [9]. Elevated venous pulsations have been implicated in the pathogenesis of white matter disease [5] (white matter disease is a major substrate of $\mathrm{VaD}$ ) and hyperdynamic venous compression leading to elevated venous pulsations has been suggested as a cause for the venous collagenosis seen in leukoaraiosis [27]. Leukoaraiosis is noted to occur in the periventricular and deep white matter, relatively sparing the cortical $U$ fibres and there are also associated changes in the deep grey matter [28]. The deep venous territory drains to within 1-2 cm of the cortex with the $U$ fibers being drained by the superficial system [29]. Therefore, the region where the pathology of $\mathrm{VaD}$ and leukoaraiosis are found is the deep venous territory suggesting hyperdynamic venous pulsation in the ST territory $(2.75$ times larger than in normal young and 1.57 times larger than in normal elderly) may be important in the causation of $\mathrm{VaD}$ as well. 
CONCLUSION

Aging and senile dementia bring about an increase in the energy stored in the pulse pressure of the arterial pressure wave, this energy is dissipated as pulsations throughout the cranial cavity. With aging the amount of CSF expelled into the vertebral cavity from the incisura is not increased indicating that there is a relative failure of the windkessel mechanism to dampen the pulsations. This leads to an increase in venous compression. In addition, senile dementia is associated with a redistribution of pulsation energy between various venous territories. The earliest manifestations of SDAT and VaD appear to occur in regions of altered venous pulsation.

\section{CONFLICT OF INTEREST STATEMENT}

We declare that we have no conflict of interest.

\section{ACKNOWLEDGMENTS}

Australian Brain Foundation and John Hunter Hospital Research Committee for granting funding for this research.

The experiments conducted in this paper comply with the current laws of the country of Australia. 


\section{REFERENCES}

1. Greitz D (1993) Cerebrospinal fluid circulation and associated intracranial dynamics: A radiologic investigation using MR imaging and radionuclide cisternography. Acta Neuroradio Suppl 34:6-23

2. Egnor M, Zheng L, Rosiello A, Gutman F, Davis R (2002) A model of pulsations in communicating hydrocephalus. Pediatr Neurosurg 36:281-303

3. Bateman GA (2000) Vascular compliance in normal pressure hydrocephalus. AJNR 21:1574-1585.

4. Bateman GA (2007) Magnetic resonance imaging quantification of compliance and collateral flow in late-onset idiopathic aqueductal stenosis: venous pathophysiology revisited. J Neurosurg 107:951-958

5. Bateman GA (2002) Pulse-wave encephalopathy: a comparative study of the hydrodynamics of leukoaraiosis and normal-pressure hydrocephalus. Neuroradiology 44: 740-748.

6. Bateman GA (2004) Pulse wave encephalopathy: a spectrum hypothesis incorporating Alzheimer's disease, vascular dementia and normal pressure hydrocephalus. Med Hypothesis 62: 182-187

7. Stivaros SM, Jackson A (2007) Changing concepts of cerebrospinal fluid hydrodynamics: role of phase-contrast magnetic resonance imaging and implications for cerebral microvascular disease. Neurotherapeutics 4: 511-522

8. Henry-Feugeas MC (2007) Alzheimer's disease in late-life dementia: A minor toxic consequence of devastating cerebrovascular dysfunction. Med Hypotheses 65: Epub ahead of print 
9. Bateman GA, Levi CR, Schofield P, Wang Y, Lovett EC (2006) Quantitative measurement of cerebral haemodynamics in early vascular dementia and Alzheimer's disease. J Clin Neurosci 13:563-568

10. Evans AJ, Iwai F, Grist TA, et al (1993) MR imaging of blood flow with a phase subtraction technique: in vitro and in vivo validation. Invest Radiol 28: 109-115.

11. Laffon E, Valli N, Latrabe V, et al (1998) A validation of a flow quantification by MR phase mapping software. Eur J Radiol 27:166-172.

12. Powell AJ, Maier SE, Chung T, et al (2000) Phase-velocity cine magnetic resonance imaging measurement of pulsatile blood flow in children and young adults: in vitro and in vivo validation. Pediatr Cardiol 21: 104-110.

13. Enzmann DR, Pelc NJ (1993) Cerebrospinal fluid flow measured by phasecontrast cine MR. AJNR 14: 1301-1307.

14. Guyton AC, Hall JE (eds) (2000) Textbook of medical physiology. $10^{\text {th }}$ ed. Saunders, Philadelphia, pp 154-155

15. O'Rourke MF, Hashimoto J (2007) Mechanical factors in arterial aging: a clinical perspective. J Am Coll Cardiol 50:1-13

16. Stoquart-ElSankari S, Baledent O, Gondry-Jouet C, Makki M, Godefroy O, Meyer ME (2007) Aging effects on cerebral blood flow and cerebrospinal fluid flows. J Cereb Blood Flow Metab 27:1563-1572

17. Czosnyka M, Czosnyka ZH, Whitfield PC, Donovan T, Picard JD. Age dependence of cerebrospinal pressure-volume compensation in patients with hydrocephalus. J Neurosurg 2001; 94:482-486

18. Kim J, Thacker NA, Bromiley PA, Jackson A (2007) Prediction of the jugular venous waveform using a model of CSF dynamics. AJNR 28:983-989 
19. Patankar T, Widjaja E, Chant H, McCollum C, Baldwin R, Jeffries S (2006) Relationship of deep white matter hyperintensities and cerebral blood flow in severe carotid artery stenosis. Eur J Neurol 13:10-6

20. Stopa EG, Berzin TM, Kim S, Song P, Kuo-LeBlanc V, Rodriguez-Wolf M et al (2001) Human choroid plexus growth factors: what are the implications for CSF dynamics in Alzheimer's disease. Exp Neurol 167:40-47

21. Uftring SJ, Chu D, Alperin N, Levin DN (2000) The mechanical state of intracranial tissues in elderly subjects studied by imaging CSF and brain pulsations. Magn Reson Imaging 18:991-996.

22. Higuchi Y, Miyakawa T, Shimoji A, et al (1987) Ultrastructural changes of blood vessels in the cerebral cortex in Alzheimer's disease. Jpn J Psychiatry Neurol 41: 283-290.

23. Kalaria RN (1996) Cerebral vessels in aging and Alzheimer's disease. Pharmacol Ther 72: 193-214.

24. Buee L, Hof PR, Delacourte A (1997) Brain microvascular changes in Alzheimer's disease and other dementias. Ann N Y Acad Sci 26: 7-24.

25. Farrall AJ, Wardlaw JM (2007) Blood-brain barrier: Ageing and microvascular disease- systemic review and meta-analysis. Neurobiol Aging In press doi:10.1016/j.neurobiolaging.2007.07.015

26. Esiri MM, Wilcock GK, Morris JH (1997) Neuropathological assessment of the lesions of significance in vascular dementia. J Neurol Neurosurg Psychiatry 63: $749-753$.

27. Moody DM, Brown WR, Challa VR, Anderson RL (1995) Periventricular venous collagenosis: association with leukoaraiosis. Radiology 194: 496-476. 
28. Henry-Feugeas MC, DeMarco G, Idy-Peretti I, Gordon-Hardy S, Fredy D, Claeys ES (2005) Age-related cerebral white matter changes and pulse-wave encephalopathy: observations with three-dimensional MRI. Magn Res Imaging 23:929-937

29. Schaller B (2004) Physiology of cerebral venous blood flow: from experimental data in animals to normal function in humans Brain Res Rev 46:243-260. 


\section{TABLE 1}

\section{Non pulsatile hemodynamics}

\begin{tabular}{|c|c|c|c|c|c|}
\hline & $\begin{array}{l}\text { Arterial } \\
\text { inflow }\end{array}$ & SSS outflow & ST outflow & $\begin{array}{l}\text { SSS outflow as a } \\
\% \text { of inflow }\end{array}$ & $\begin{array}{l}\text { ST outflow as a } \% \\
\text { of inflow }\end{array}$ \\
\hline & $\mathrm{mL} / \mathrm{min}$ & $\mathrm{mL} / \mathrm{min}$ & $\mathrm{mL} / \mathrm{min}$ & $\%$ & $\%$ \\
\hline Normal young & $900(160)$ & $400(80)$ & $120(40)$ & $45(7)$ & $14(3)$ \\
\hline \multicolumn{6}{|l|}{$\mathrm{n}=12$} \\
\hline Normal elderly & $710(110)^{*}$ & $310(60)^{*}$ & $90(30)^{*}$ & $44(8)$ & $13(4)$ \\
\hline \multicolumn{6}{|l|}{$\mathrm{n}=12 \quad(\mathrm{SD})$} \\
\hline Vascular & $790(270)$ & $290(70)$ & $90(30)$ & $38(5)^{*}$ & $12(3)$ \\
\hline $\mathrm{n}=12 \quad(\mathrm{SD})$ & & & & & \\
\hline Alzheimer & $580(110)^{*}$ & $260(50)^{*}$ & $80(10)$ & $46(8)$ & $13(3)$ \\
\hline
\end{tabular}

Note.- SSS, superior sagittal sinus; $\mathrm{ST}$, straight sinus; $\mathrm{mL} / \mathrm{min}$, milliliters per minute; $\mathrm{SD}$, standard deviation; ${ }^{*}, \mathrm{p}$ value $<0.05$. 


\section{TABLE 2}

\section{Pulsatile hemodynamics}

\begin{tabular}{|c|c|c|c|c|c|}
\hline & Arterial pulse & $\begin{array}{l}\text { SSS } \\
\text { pulse }\end{array}$ & $\begin{array}{c}\text { ST } \\
\text { pulse }\end{array}$ & $\begin{array}{l}\text { Incisural } \\
\text { pulse }\end{array}$ & $\begin{array}{c}\text { Basal venous } \\
\text { pulse }\end{array}$ \\
\hline & $\mu \mathrm{L}$ & $\mu \mathrm{L}$ & $\mu \mathrm{L}$ & $\mu \mathrm{L}$ & $\mu \mathrm{L}$ \\
\hline Normal young & $890(270)$ & $200(90)$ & $40(15)$ & $310(70)$ & $430(160)$ \\
\hline $\mathrm{n}=12 \quad(\mathrm{SD})$ & & & & & \\
\hline Normal elderly & $1330(370)^{*}$ & $310(120)^{*}$ & $70(20)^{*}$ & $280(110)$ & $670(210)^{*}$ \\
\hline $\mathrm{n}=12 \quad(\mathrm{SD})$ & & & & & \\
\hline Vascular & $1650(380)^{*}$ & $310(80)$ & $110(50)^{*}$ & $460(110)^{*}$ & $770(310)$ \\
\hline $\mathrm{n}=12 \quad(\mathrm{SD})$ & & & & & \\
\hline Alzheimer & $1150(380)$ & $310(150)$ & $70(30)$ & $360(90)$ & $410(270)^{*}$ \\
\hline$n=12 \quad(S D)$ & & & & & \\
\hline
\end{tabular}

Note.-; SSS, superior sagittal sinus; ST, straight sinus; $\mu \mathrm{L}$, microlitres; SD, standard deviation; ${ }^{*}$, p value $<0.05$. 